\title{
Tea consumption and oxidative stress: a cross-sectional analysis of 889 premenopausal women from the Sister Study
}

\author{
Dongyu Zhang ${ }^{1}$, Kelly Ferguson ${ }^{2}$, Melissa A. Troester ${ }^{1}$, Jeannette T. Bensen ${ }^{1}$, Jianwen Cai ${ }^{3}$, \\ Ginger L. Milne ${ }^{4}$, Dale P. Sandler ${ }^{2}$ and Hazel B. Nichols ${ }^{1 *}$ \\ ${ }^{1}$ Department of Epidemiology, University of North Carolina at Chapel Hill Gillings School of Global Public Health, \\ Chapel Hill, NC 27599, USA \\ ${ }^{2}$ Epidemiology Branch, National Institute of Environmental Health Sciences, Research Triangle Park, NC 27709, USA \\ ${ }^{3}$ Department of Biostatistics, University of North Carolina at Chapel Hill Gillings School of Global Public Health, \\ Chapel Hill, NC 27599, USA \\ ${ }^{4}$ Eicosanoid Core Laboratory, Division of Clinical Pharmacology, Vanderbilt University Medical Center, Nashville, TN 37232, \\ USA \\ (Submitted 22 May 2018 - Final revision received 15 October 2018 - Accepted 7 December 2018 - First published online 24 January 2019)
}

\section{Abstract}

In experimental and clinical studies, green or black tea consumption has been shown to reduce oxidative stress. However, these studies involved high levels of tea consumption and may not reflect patterns in the general population. Here, we examined the association between black or green tea consumption and oxidative stress in a cross-sectional study of 889 premenopausal US women aged 35-54 years. Tea consumption was measured using the Block-98 FFQ. Urinary 8-iso-PGF $\mathrm{PG}_{2 \alpha}\left(\mathrm{F}_{2}\right.$-IsoP) and 2,3-dinor-5,6-dihydro-15- $\mathrm{F}_{2 \mathrm{t}}$-isoprostane (15- $\mathrm{F}_{2 \mathrm{t}}$-IsoPM) were used as biomarkers of oxidative stress. These compounds were measured by MS and normalised to creatinine. Linear regression was used to calculate the geometric mean differences (GMD) and $95 \% \mathrm{CI}$ for log-transformed urinary $\mathrm{F}_{2}$-IsoP or $15-\mathrm{F}_{2 \mathrm{t}}$-IsoP-M in relation to black or green tea consumption. We further examined whether adjusting for caffeine impacted associations between tea and oxidative stress. Geometric means of urinary $\mathrm{F}_{2}$-IsoP and $15-\mathrm{F}_{2 \mathrm{t}} \mathrm{Is}$ IsoP-M were 1.44 (95\% CI 1.39, 1.49) and 0.71 (95\% CI $\left.0.69,0.73\right) \mathrm{ng} / \mathrm{mg} \mathrm{creatinine}$, respectively. Overall, green tea consumption was not associated with urinary $\mathrm{F}_{2}$-IsoP or $15-\mathrm{F}_{2 \mathrm{t}}$-IsoP-M. High-level black tea consumption $(\geq 5$ cups/week compared with 0) was associated with higher $15-\mathrm{F}_{2 t}-\mathrm{IsoP}-\mathrm{M}$ concentrations (adjusted GMD $=0 \cdot 10,95 \% \mathrm{CI} 0 \cdot 02-0.19$ ) but not $\mathrm{F}_{2-}$ IsoP. Adjusting for caffeine nullified the association between black tea and $15-\mathrm{F}_{2 \mathrm{t}}$-IsoP-M. Our findings do not support the hypothesis that dietary tea consumption is inversely associated with oxidative stress.

Key words: Tea: Oxidative stress: Epidemiology: Women's health

Oxidative stress describes an imbalance of oxidant/antioxidant networks that results in the disruption of redox signalling and/ or molecular damage ${ }^{(1-3)}$. In humans, persistent oxidative stress can lead to oxidation of lipids, alteration of protein function and mutation of $\mathrm{DNA}^{(4-7)}$. These disruptions may contribute to the pathogenesis of cancer, diabetes and neurodegenerative disease ${ }^{(8-10)}$. To detect increases in oxidative stress, oxidation products of lipids are often used as biomarkers ${ }^{(11)}$. Urinary 8 -iso- $\mathrm{PGF}_{2 \alpha}\left(\mathrm{F}_{2}\right.$-IsoP) and its primary metabolite, 2,3-dinor-5,6dihydro- $15-\mathrm{F}_{2 \mathrm{t}}$-isoprostane $\left(15-\mathrm{F}_{2 \mathrm{t}}-\mathrm{IsoP}-\mathrm{M}\right)$, are stable biomarkers of lipid peroxidation ${ }^{(12,13)}$. Several studies have reported higher levels of specific urinary $\mathrm{F}_{2}$-IsoP in medical conditions including cancer and $\mathrm{CVD}^{(14-16)}$

Tea is a popular and accessible beverage worldwide ${ }^{(17)}$ and may have beneficial health effects for diabetes, CVD and cancer $^{(18-20)}$. Green tea and black tea contain polyphenols ${ }^{(21)}$ that have potential antioxidant properties ${ }^{(22)}$. For example, the polyphenol epigallocatechin-3-gallate (EGCG) is a natural antioxidant found in green and black tea ${ }^{(23,24)}$. To experimentally study the anti-oxidative effects of tea (green tea, black tea and green tea extract ${ }^{(25-28)}$, participants were assigned to high levels of tea consumption for a short period of time (e.g. 4 cups of green tea/d for 8 weeks or placebo beverage). Participants in the intervention group drinking green tea had lower levels of blood malondialdehyde (MDA), a biomarker of oxidative stress $^{(25)}$. Participants assigned to receiving one capsule $(379 \mathrm{mg})$ of green tea extract per $\mathrm{d}$ for 3 months had a higher total antioxidant status compared with placebo ${ }^{(26)}$. A third study reported that black tea consumption ( $4 \mathrm{cups} / \mathrm{d}$ for 6 months) reduced lipid peroxidation among thirty-four female former smokers ${ }^{(28)}$. These findings support a potential inverse association between tea consumption and oxidative stress.

Abbreviations: $15-\mathrm{F}_{2 \mathrm{t}}$-IsoP-M, 2,3-dinor-5,6-dihydro-15- $\mathrm{F}_{2} \mathrm{t}$-isoprostane; $\mathrm{F}_{2}$-IsoP, 8-iso-PGF ${ }_{2 \alpha}$

* Corresponding author: Dr H. B. Nichols, email hazel.nichols@unc.edu 
However, in the general population, tea consumption patterns may reflect longer durations of lower consumption levels. It is unknown whether these patterns also translate to benefits for oxidative stress. Here, we conducted a cross-sectional analysis of 889 premenopausal women to examine the association between tea consumption and oxidative stress.

\section{Methods}

Data for this analysis come from the National Institute of Environmental Health Sciences (NIEHS) Sister Study. Study participants ( $n$ 50 884) aged 35-74 years were enroled between 2003 and 2009 across the USA and Puerto Rico ${ }^{(29)}$. To be eligible, participants had at least one sister who had been diagnosed with breast cancer, but no personal history of breast cancer. All participants provided written consent at enrolment. Study protocols were approved by the Institutional Review Board of the NIEHS, the National Institutes of Health and the Copernicus Group.

\section{Population for analysis}

Within the Sister Study, 1367 women were identified (456 cases, 911 controls) for a nested case-control study investigating oxidative stress and breast cancer risk among premenopausal women. To be eligible for the nested case assigned control study, participants had to meet the following criteria: aged 54 and younger, premenopausal status (at least one menstrual cycle in the previous 12 months or hysterectomy with $\geq 1$ ovary conserved) and have an available blood and urine sample from enrolment. Controls were matched to cases with a ratio of $2: 1$ on the basis of age and enrolment year and were free of breast cancer at the time of their matched case's diagnosis. For this analysis, information from the 911 controls was used. We further excluded women missing data of black and green tea consumption levels ( $n$ 22), which yielded 889 participants for this study.

\section{Oxidative stress measurement}

At Sister Study enrolment, participants self-collected approximately $60 \mathrm{ml}$ of first-morning void urine in a study-provided collection cup ${ }^{(29)}$. Participants refrigerated the samples without preservative until they were picked up by study examiners who shipped the samples on ice to the study repository ${ }^{(30)}$. On receipt, urine samples were aliquoted and stored at $-80^{\circ} \mathrm{C}$. In 2012, samples were retrieved and urinary concentrations of $\mathrm{F}_{2}$-IsoP and $15-\mathrm{F}_{2 \mathrm{t}}$-IsoP-M were measured using $\mathrm{GC} /$ negative ion chemical ionisation MS at the Eicosanoid Core Laboratory at Vanderbilt University Medical Center. The mean storage time of urinary samples was 8.3 years. Protocols for chemical analysis and procedures have been described in detail ${ }^{(31-34)}$. A total of seventy-seven batches were run; each batch contained eighteen samples from study subjects (twelve controls and six cases) and two quality control (QC) samples. The CV for QC duplicates was $16 \cdot 0$ and $12.5 \%$ for $\mathrm{F}_{2}$-IsoP and $15-\mathrm{F}_{2 \mathrm{t}}$-IsoP-M, respectively ${ }^{(35)}$. Urinary levels of $\mathrm{F}_{2}$-IsoP and $15-\mathrm{F}_{2 \mathrm{t}}$-IsoP-M were adjusted for creatinine (ng/mg creatinine) to correct for urine diluteness.

\section{Exposure and covariate measurement}

During an enrolment home visit, trained examiners measured height and weight without shoes. These measurements were taken three times and values were rounded to the nearest quarter inch for height and whole pound for weight ${ }^{(36)}$. BMI was calculated as weight $(\mathrm{kg}) /$ height $(\mathrm{m})^{2}$. Black and green tea consumption during the past 12 months was measured by the self-administered Block 98 FFQ at study enrolment ${ }^{(37)}$. Within the FFQ, participants reported their frequency of tea consumption and the cups consumed each time ${ }^{(29)}$. Frequency was reported at nine levels, ranging from 'never' to 'everyday'. Participants reported how many cups of tea they consumed each time as ' 1 cup, 2 cups, 3-4 cups or 5 or more cups'. Regular (non-decaffeinated) coffee consumption was measured using the same methods described above. Healthy eating index, dietary fruit and vegetable intake and dietary $\beta$-carotene, vitamin $\mathrm{C}$, and vitamin $\mathrm{E}$ intake were obtained via information collected in self-administered FFQ. Total energy intake and caffeine from beverages (soda and black tea) and dietary sources were calculated from the FFQ by NutritionQuest $^{(38)}$. We assigned caffeine levels to coffee (regular and decaffeinated) and green tea on the basis of data from USDA Food Composition Databases ${ }^{(39,40)}$. Each cup of regular coffee was assigned $95.2 \mathrm{mg}$ caffeine, each cup of decaffeinated coffee $2 \mathrm{mg}$ caffeine, and each cup of green tea $24.8 \mathrm{mg}$ caffeine. On average, one cup of black tea contains $47.2 \mathrm{mg}$ caffeine $^{(39)}$.

A validated series of questions were used for measurement of physical activity ${ }^{(41,42)}$. Weekly energy expenditures at enrolment were calculated as metabolic equivalents (MET), and total physical activity was calculated by summing the MET-h/week of all sports, physical exercise and daily activity self-reported at enrolment. Participants were also asked for information about the total annual income from all household members and the highest level of school they had completed as well as their age and race/ethnicity.

\section{Statistical analysis}

Tea consumption was categorised into four levels $(0,<1,1-<5$ and $\geq 5$ cups/week). The consumption value was obtained by multiplying the frequency of consumption (times/week) and serving size (cups consumed each time) together. Cut-off points were determined based on the distribution of tea consumption among women who reported drinking black tea to approximate tertiles. Non-consumers were identified $a$ priori as the reference group. The same cut-off points were used for green tea consumption for consistency. Tea consumers with missing serving size information (twenty-two for black tea, twenty-four for green tea) were assigned a serving size of one cup per serving (the most common serving size for black (56.7\%) and green (76.0\%) tea consumption). For coffee consumption, one and two cups per serving were about equally common $(37.0$ and $38.5 \%$, respectively), and coffee consumers with missing 
serving size information ( $n$ 13) were also assigned as drinking one cup per serving. Coffee consumption was categorised into four levels: $0,<10,10-<15$ and $\geq 15$ cups/week. Caffeine intake was categorised based on approximate quartiles $(<33.9$, $33 \cdot 9-<111 \cdot 2,111 \cdot 2-<205 \cdot 2$ and $\geq 205 \cdot 2 \mathrm{mg} / \mathrm{d}$ ). BMI categories were defined based on WHO guidelines as underweight/ normal weight $\left(<24.9 \mathrm{~kg} / \mathrm{m}^{2}\right)$, pre-obesity $\left(25.0-29.9 \mathrm{~kg} / \mathrm{m}^{2}\right)$, obesity class I $\left(30 \cdot 0-34 \cdot 9 \mathrm{~kg} / \mathrm{m}^{2}\right)$, obesity class II $(35 \cdot 0-$ $\left.39.9 \mathrm{~kg} / \mathrm{m}^{2}\right)$ and obesity class III $\left(\geq 40 \mathrm{~kg} / \mathrm{m}^{2}\right)^{(43)}$. The healthy eating index, dietary fruit and vegetable intake and dietary $\beta$-carotene, vitamin $\mathrm{C}$, vitamin $\mathrm{E}$, total energy intake and physical activity were categorised to approximate quartiles.

Geometric means and $95 \% \mathrm{CI}$ of urinary $\mathrm{F}_{2}$-IsoP and $15-\mathrm{F}_{2 \mathrm{t}^{-}}$ IsoP-M were calculated for each level of tea consumption and by other covariates. The distribution of urinary $\mathrm{F}_{2}$-IsoP and 15$\mathrm{F}_{2 \mathrm{t}}$-IsoP-M concentrations was right skewed; thus, a natural log transformation was applied for these biomarkers to approximate normality. Univariate geometric mean difference and adjusted geometric mean difference (aGMD) and 95\% CI of urinary $\mathrm{F}_{2}$-IsoP and $15-\mathrm{F}_{2 \mathrm{t}}$ IsoP-M were calculated using linear regression of the natural log-transformed values. To calculate aGMD of $\mathrm{F}_{2}$-IsoP or $15-\mathrm{F}_{2 \mathrm{t}}$-IsoP-M across tea consumption levels, the linear regression model adjusted for age $(35-<40$, $40-<45,45-<50$ and $\geq 50$ years), race (non-Hispanic white, non-Hispanic black and other), BMI (BMI, $<25,25-<30$, $30-<35,35-<40$ and $\geq 40 \mathrm{~kg} / \mathrm{m}^{2}$ ), education (high school or less, some college or undergraduate and graduate school), annual income $(0-<\$ 50000, \$ 50000-<\$ 100000$ and $\geq \$ 100000)$, smoking status (never, former and current), healthy eating index $(<53,53-<63,63-<72$ and $\geq 72)$, dietary fruit $(<0 \cdot 6,0 \cdot 6-<1 \cdot 1,1 \cdot 1-<2$ and $\geq 2$ servings/d) and vegetable intake ( $<1 \cdot 6,1 \cdot 6-<2 \cdot 7,2 \cdot 7-<4 \cdot 3$ and $\geq 4 \cdot 3$ servings/d), dietary $\beta$-carotene $(<2427 \cdot 2,2427 \cdot 2-<4106 \cdot 1,4106 \cdot 1-<6942 \cdot 3$ and $\geq 6942 \cdot 3 \mu \mathrm{g} / \mathrm{d}), \quad$ vitamin $\quad \mathrm{C} \quad(<55 \cdot 3, \quad 55 \cdot 3-<84 \cdot 1$, $84.1-<121.9 \geq 121.9 \mathrm{mg} / \mathrm{d})$ and vitamin $\mathrm{E}$ intake $(<5.6$, $5 \cdot 6-<7 \cdot 6,7 \cdot 6-<10 \cdot 1 \geq 10 \cdot 1 \mathrm{mg} / \mathrm{d})$, total energy intake ( $<5148 \cdot 0$, $5148 \cdot 0-<6396 \cdot 1,6396 \cdot 1-<8263 \cdot 0$ and $\geq 8263 \cdot 0 \mathrm{~kJ} / \mathrm{d})$ and physical activity $(<28 \cdot 11,28.11-<44.16,44.16-<65.99, \geq 65.99$ MET-h/week) were used as potential confounders ${ }^{(44)}$. We also evaluated the impact of additional adjustment for caffeine $(<33 \cdot 9,33 \cdot 9-<111 \cdot 2,111 \cdot 2-<205 \cdot 2 \text { and } \geq 205 \cdot 2 \mathrm{mg} / \mathrm{d})^{(45,46)}$. The assumptions of the linear regression (linearity, independence, multivariate normality and homoscedasticity) were examined by scatterplots of urinary $\mathrm{F}_{2}$-IsoP or $15-\mathrm{F}_{2 \mathrm{t}}$-IsoP-M $v$. tea consumption and plots of the residuals $v$. fitted values of the regression model; results did not suggest that assumptions were violated.

Subgroup analyses were conducted to address potential effect modification of associations between tea and $\mathrm{F}_{2}$-IsoP or $15-\mathrm{F}_{2 \mathrm{t}}$-IsoP-M according to overweight (BMI $<25 \mathrm{~kg} / \mathrm{m}^{2} v$. BMI $\geq 25 \mathrm{~kg} / \mathrm{m}^{2}$ ) and regular coffee consumption (drinker $v$. nondrinker). Interaction terms between tea consumption and the covariates were included in multivariable linear regressions, and log-likelihood ratio tests were used to assess whether the interaction terms were statistically significant.

We did not adjust for multiple comparisons as our analysis was hypothesis driven ${ }^{(47-49)}$. Two-sided $P$ values $<0.05$ were considered to be statistically significant. All statistical analyses were conducted with Sister Study data release 6.0 using Stata 13.0 (StataCorp, LLP).

\section{Results}

Of the 889 participants in our analysis, the average age at baseline was 47.28 (SD 4.45) and the majority were nonHispanic white $(87 \cdot 3 \%)$. The geometric means of urinary $\mathrm{F}_{2^{-}}$ IsoP and $15-\mathrm{F}_{2 \mathrm{t}}$-IsoP-M were $1.44(95 \% \mathrm{CI} 1.39,1.49)$ and 0.71 (95\% CI $0.69,0.73) \mathrm{ng} / \mathrm{mg}$ creatinine, respectively. Table 1 presents geometric means and mean differences of urinary $\mathrm{F}_{2^{-}}$ IsoP and $15-\mathrm{F}_{2 \mathrm{t}}$-IsoP-M according to participant characteristics. Both $\mathrm{F}_{2}$-IsoP and $15-\mathrm{F}_{2 \mathrm{t}}$-IsoP-M decreased slightly as age increased, but the differences were not statistically significant. Average $\mathrm{F}_{2}$-IsoP and $15-\mathrm{F}_{2 \mathrm{t}}$-IsoP-M levels among nonHispanic black women were lower compared with white women. Levels of both $\mathrm{F}_{2}$-IsoP and $15-\mathrm{F}_{2 \mathrm{t}}$-IsoP-M were positively associated with BMI and inversely associated with higher income and physical activity. Current smokers had higher levels of both $\mathrm{F}_{2}$-IsoP and $15-\mathrm{F}_{2 \mathrm{t}}$-IsoP-M compared with never smokers, but associations were statistically significant only for $15-\mathrm{F}_{2 \mathrm{t}}$-IsoP-M. Inverse but non-significant associations with education were also observed for $15-\mathrm{F}_{2 \mathrm{t}^{-}}$ IsoP-M but not $\mathrm{F}_{2}$-IsoP. High-level coffee consumption $(\geq 15$ cups/week) was not associated with $\mathrm{F}_{2}$-IsoP or $15-\mathrm{F}_{2 \mathrm{t}}$-IsoP-M. Total energy intake was positively associated with $\mathrm{F}_{2}$-IsoP or $15-\mathrm{F}_{2 \mathrm{t}}$-IsoP-M. Associations with the healthy eating index, vegetable intake and vitamin $\mathrm{C}$ were not statistically significant for $\mathrm{F}_{2}$-IsoP and $15-\mathrm{F}_{2 \mathrm{t}}$-IsoP-M. Higher fruit intake and dietary $\beta$-carotene were inversely associated with $\mathrm{F}_{2}$-IsoP and $15-\mathrm{F}_{2 \mathrm{t}}$-IsoP-M, respectively, but not with both markers. Dietary vitamin $\mathrm{E}$ appeared inversely associated with both biomarkers, but only estimates of $15-\mathrm{F}_{2 \mathrm{t}}-\mathrm{IsoP}-\mathrm{M}$ were statistically significant.

Associations between black and green tea consumption and caffeine intake with urinary oxidative stress measures are shown in Table 2. Black tea consumption was more common than green tea consumption; $18.6 \%$ of women reported never drinking black tea, while $45.9 \%$ of women reported never drinking green tea. The highest level of consumption, $\geq 5$ cups/week, was reported by 24.9 and $7.6 \%$ of women for black and green tea consumption, respectively. Overall, black and green tea consumption were not associated with $\mathrm{F}_{2}$-IsoP levels (Table 2). However, mean concentrations of $15-\mathrm{F}_{2 \mathrm{t}}$-IsoP-M were higher for black tea consumption of 5 cups/week or more compared with 0 cups/week (aGMD $=0 \cdot 10,95 \%$ CI $0 \cdot 02,0 \cdot 19)$. High-level green tea consumption ( $\geq 5$ cups/week compared with 0) was not significantly associated with $15-\mathrm{F}_{2 \mathrm{t}}-\mathrm{IsoP}-\mathrm{M} \quad(\mathrm{aGMD}=0.09$, $95 \% \mathrm{CI}-0 \cdot 02,0 \cdot 20)$.

Caffeine intake was not associated with $\mathrm{F}_{2}$-IsoP. As compared with the lowest quartile $(<33.9 \mathrm{mg} / \mathrm{d})$, higher levels of caffeine intake were positively associated with 15 $\mathrm{F}_{2 \mathrm{t}}$-IsoP-M, but there was no consistent increase across quartiles. Additional adjustment for caffeine intake attenuated the association between black tea and $15-\mathrm{F}_{2 \mathrm{t}}-\mathrm{IsoP}-\mathrm{M}$ towards the null (Table 2). Associations between tea 
Table 1. Characteristics of study participants and estimates of urinary 8-iso- $\mathrm{PGF}_{2 a}\left(\mathrm{~F}_{2}\right.$-IsoP) or 2,3-dinor-5,6-dihydro-15- $\mathrm{F}_{2} \mathrm{t}$-isoprostane $\left(15-\mathrm{F}_{2 t}-\mathrm{IsoP}_{\mathrm{S}} \mathrm{M}\right)$ by covariates ${ }^{*}$ (Numbers and percentages; geometric means (GM) and $95 \%$ confidence intervals)

\begin{tabular}{|c|c|c|c|c|c|c|c|c|c|c|c|c|c|c|}
\hline \multirow[b]{2}{*}{ Characteristics } & \multicolumn{2}{|c|}{$n 889$} & \multicolumn{6}{|c|}{$\mathrm{F}_{2}$-IsoP } & \multicolumn{6}{|c|}{$15-F_{2 t}$-IsoP-M } \\
\hline & $n$ & $\%$ & GM & $95 \% \mathrm{Cl} \dagger$ & uGMD & $95 \% \mathrm{Cl}$ & aGMD & $95 \% \mathrm{Cl} \S$ & GM & $95 \% \mathrm{Cl} \dagger$ & uGMD & $95 \% \mathrm{Cl} \ddagger$ & aGMD & $95 \% \mathrm{Cl}$ \\
\hline \multicolumn{15}{|l|}{ Age (years) } \\
\hline $35-<40$ & 61 & $6 \cdot 9$ & 1.61 & $1.41,1.83$ & \multicolumn{2}{|r|}{ Ref. } & \multicolumn{2}{|r|}{ Ref. } & 0.75 & $0.67,0.83$ & \multicolumn{2}{|r|}{ Ref. } & \\
\hline $40-<45$ & 177 & 19.9 & 1.43 & $1.33,1.53$ & -0.13 & $-0.28,0.02$ & -0.09 & $-0.24,0.05$ & 0.72 & $0.67,0.77$ & -0.04 & $-0.17,0.09$ & \multicolumn{2}{|r|}{$\begin{array}{l}\text { Ref. } \\
-0.13,0.11\end{array}$} \\
\hline $45-<50$ & 377 & $42 \cdot 4$ & 1.44 & $1.36,1.51$ & -0.11 & $-0.25,0.03$ & -0.07 & $-0.20,0.07$ & 0.73 & $0.70,0.76$ & -0.03 & $-0.15,0.09$ & 0.00 & $-0.11,0.12$ \\
\hline$\geq 50$ & 274 & $30 \cdot 8$ & 1.41 & $1.32,1.51$ & -0.13 & $-0.28,0.01$ & -0.08 & $-0.22,0.06$ & 0.68 & $0.65,0.72$ & -0.10 & $-0.23,0.02$ & -0.06 & $-0 \cdot 18,0.05$ \\
\hline \multicolumn{15}{|l|}{ Race } \\
\hline Non-Hispanic white & 776 & $87 \cdot 3$ & 1.45 & $1.40,1.51$ & \multicolumn{2}{|r|}{ Ref. } & \multicolumn{2}{|r|}{ Ref. } & 0.72 & $0.69,0.74$ & \multicolumn{2}{|r|}{ Ref. } & \multicolumn{2}{|r|}{ Ref. } \\
\hline Non-Hispanic black & 56 & $6 \cdot 3$ & 1.25 & $1.09,1.44$ & -0.14 & $-0.28,0.00$ & -0.23 & $-0.37,-0.09$ & 0.65 & $0.58,0.74$ & -0.09 & $-0.21,0.03$ & -0.18 & $-0.29,-0.06$ \\
\hline Other & 57 & $6 \cdot 4$ & 1.42 & $1.27,1.60$ & -0.02 & $-0.16,0.12$ & -0.01 & $-0.15,0.13$ & 0.73 & $0.65,0.81$ & 0.01 & $-0.11,0.13$ & 0.01 & $-0.10,0.12$ \\
\hline \multicolumn{15}{|l|}{ BMI $\left(\mathrm{kg} / \mathrm{m}^{2}\right)$} \\
\hline$<25$ & 406 & $45 \cdot 7$ & 1.29 & $1.23,1.36$ & \multicolumn{2}{|r|}{ Ref. } & & Ref. & 0.63 & $0.60,0.65$ & & Ref. & & Ref. \\
\hline $25-<30$ & 246 & $27 \cdot 7$ & 1.46 & $1.38,1.55$ & 0.13 & $0.04,0.21$ & $0 \cdot 11$ & $0.03,0.20$ & 0.70 & $0.67,0.74$ & 0.12 & $0.05,0.19$ & 0.10 & $0.03,0.17$ \\
\hline $30-<35$ & 122 & 13.7 & 1.50 & $1.37,1.65$ & 0.15 & $0.05,0.26$ & $0 \cdot 10$ & $-0.01,0.20$ & 0.79 & $0.74,0.85$ & 0.24 & $0.16,0.33$ & 0.17 & $0.08,0.26$ \\
\hline $35-<40$ & 67 & 7.5 & 1.71 & $1.49,1.97$ & 0.27 & $0.14,0.40$ & $0 \cdot 19$ & $0.05,0.32$ & 0.94 & $0.84,1.05$ & 0.41 & $0.30,0.52$ & 0.36 & $0.25,0.47$ \\
\hline$\geq 40$ & 47 & $5 \cdot 3$ & $2 \cdot 23$ & $1.90,2.63$ & 0.54 & $0.39,0.69$ & 0.46 & $0.30,0.62$ & $1 \cdot 17$ & $1.06,1.30$ & 0.63 & $0.51,0.76$ & 0.57 & $0.44,0.70$ \\
\hline Missing & 1 & 0.1 & 6.08 & - & & & & & 1.85 & - & & & & \\
\hline Education level & & & & & & & & & & & & & & \\
\hline High school or less & 107 & $12 \cdot 0$ & 1.57 & $1.41,1.76$ & & Ref. & & Ref. & 0.84 & $0.77,0.91$ & & Ref. & & Ref. \\
\hline Some college or undergraduate & 543 & $61 \cdot 1$ & 1.42 & $1.36,1.48$ & -0.09 & $-0.20,0.02$ & 0.02 & $-0.09,0.14$ & 0.72 & $0.69,0.75$ & -0.14 & $-0.24,-0.05$ & -0.03 & $-0.12,0.06$ \\
\hline Graduate school & 239 & $26 \cdot 9$ & 1.42 & $1.33,1.52$ & -0.10 & $-0.22,0.03$ & 0.06 & $-0.07,0.18$ & 0.65 & $0.62,0.69$ & -0.25 & $-0.35,-0.14$ & -0.09 & $-0.19,0.02$ \\
\hline Annual household income (\$) & & & & & & & & & & & & & & \\
\hline$<50000$ & 144 & $16 \cdot 2$ & 1.64 & $1.50,1.80$ & & Ref. & & Ref. & 0.83 & $0.76,0.90$ & & Ref. & & Ref. \\
\hline $50000-<100000$ & 364 & $40 \cdot 9$ & 1.54 & $1.46,1.63$ & -0.05 & $-0.15,0.04$ & 0.00 & $-0.10,0.10$ & 0.76 & $0.72,0.79$ & -0.08 & $-0.17,0.00$ & 0.00 & $-0.08,0.08$ \\
\hline$\geq 100000$ & 360 & 40.5 & 1.29 & $1.23,1.35$ & -0.24 & $-0.34,-0.14$ & -0.13 & $-0.23,-0.02$ & 0.63 & $0.60,0.66$ & -0.27 & $-0.35,-0.18$ & -0.09 & $-0.18,-0.01$ \\
\hline Missing & 21 & $2 \cdot 4$ & 1.11 & $0.90,1.38$ & & & & & 0.76 & $0.63,0.91$ & & & & \\
\hline Smoking history & & & & & & & & & & & & & & \\
\hline Never & 545 & $61 \cdot 3$ & 1.41 & $1.36,1.47$ & & Ref. & & Ref. & 0.70 & $0.67,0.72$ & & Ref. & & Ref. \\
\hline Former & 267 & $30 \cdot 0$ & 1.42 & $1.33,1.52$ & -0.01 & $-0.09,0.07$ & -0.01 & $-0.08,0.07$ & 0.70 & $0.67,0.74$ & 0.01 & $-0.06,0.07$ & 0.00 & $-0.07,0.06$ \\
\hline Current & 77 & $8 \cdot 7$ & 1.69 & $1.50,1.92$ & 0.16 & $0.03,0.29$ & 0.04 & $-0.09,0.17$ & 0.88 & $0.79,0.97$ & 0.24 & $0.14,0.35$ & 0.10 & $0.00,0.21$ \\
\hline Regular (not decaffeinated) coffee & onsum & tion $(\mathrm{cl}$ & s/week & & & & & & & & & & & \\
\hline 0 & 279 & 31.4 & 1.53 & $1.44,1.63$ & & Ref. & & Ref. & 0.73 & $0.69,0.78$ & & Ref. & & Ref. \\
\hline$<10$ & 257 & 28.9 & 1.37 & $1.28,1.46$ & -0.12 & $-0.21,-0.03$ & -0.07 & $-0.15,0.02$ & 0.69 & $0.66,0.73$ & -0.06 & $-0.13,0.02$ & 0.00 & $-0.07,0.07$ \\
\hline $10-<15$ & 203 & $22 \cdot 8$ & 1.37 & $1.28,1.48$ & -0.12 & $-0.22,-0.03$ & -0.09 & $-0.19,0.00$ & 0.69 & $0.64,0.73$ & -0.06 & $-0.15,0.02$ & -0.05 & $-0.12,0.03$ \\
\hline$\geq 15$ & 143 & $16 \cdot 1$ & 1.49 & $1.36,1.62$ & -0.06 & $-0.16,0.05$ & -0.04 & $-0.14,0.07$ & 0.76 & $0.71,0.81$ & 0.03 & $-0.06,0.12$ & 0.04 & $-0.05,0.12$ \\
\hline Missing & 7 & 0.8 & 1.39 & $0.97,2.00$ & & & & & 0.63 & $0.44,0.92$ & & & & \\
\hline Total energy intake $(\mathrm{kJ} / \mathrm{d})$ & & & & & & & & & & & & & & \\
\hline$<5148.0$ & 222 & $25 \cdot 0$ & 1.41 & $1.32,1.51$ & & Ref. & & Ref. & 0.70 & $0.66,0.75$ & & Ref. & & Ref. \\
\hline $5148 \cdot 0-<6396 \cdot 1$ & 223 & $25 \cdot 1$ & 1.40 & $1.31,1.50$ & -0.02 & $-0.12,0.08$ & 0.02 & $-0.09,0.13$ & 0.68 & $0.64,0.72$ & -0.03 & $-0.12,0.05$ & 0.03 & $-0.06,0.12$ \\
\hline $6396 \cdot 1-<8263.0$ & 221 & 24.9 & 1.46 & $1.36,1.56$ & 0.03 & $-0.07,0.12$ & $0 \cdot 10$ & $-0.03,0.22$ & 0.74 & $0.69,0.78$ & 0.05 & $-0.04,0.13$ & 0.12 & $0.02,0.22$ \\
\hline$\geq 8263.0$ & 223 & $25 \cdot 0$ & 1.48 & $1.38,1.58$ & 0.02 & $-0.07,0.12$ & 0.18 & $0.03,0.32$ & 0.73 & $0.69,0.78$ & 0.05 & $-0.04,0.13$ & 0.16 & $0.04,0.28$ \\
\hline Physical activity (MET-h/week) & & & & & & & & & & & & & & \\
\hline$<28 \cdot 11$ & 221 & 24.9 & 1.68 & $1.57,1.81$ & & Ref. & & Ref. & 0.82 & $0.77,0.87$ & & Ref. & & Ref. \\
\hline $28 \cdot 11-<44 \cdot 16$ & 221 & 24.9 & 1.40 & $1.31,1.49$ & -0.18 & $-0.27,-0.08$ & -0.12 & $-0.22,-0.03$ & 0.70 & $0.66,0.74$ & -0.16 & $-0.25,-0.08$ & $-0 \cdot 10$ & $-0.18,-0.02$ \\
\hline $44.16-<65.99$ & 221 & $24 \cdot 9$ & 1.43 & $1.34,1.53$ & -0.17 & $-0.27,-0.07$ & -0.09 & $-0.18,0.01$ & 0.70 & $0.66,0.74$ & -0.17 & $-0.25,-0.09$ & -0.10 & $-0.18,-0.02$ \\
\hline$\geq 65.99$ & 221 & 24.9 & 1.27 & $1 \cdot 19,1.36$ & -0.29 & $-0.39,-0.20$ & -0.16 & $-0.25,-0.06$ & 0.65 & $-0.61,0.69$ & -0.24 & $-0.33,-0.16$ & -0.12 & $-0.20,-0.04$ \\
\hline Missing & 5 & 0.4 & 1.34 & $0.86,2.09$ & & & & & 0.76 & $0.54,1.07$ & & & & \\
\hline
\end{tabular}


Bs British Journal of Nutrition

\begin{tabular}{|c|c|c|c|c|c|c|c|c|c|c|c|c|c|c|}
\hline \multirow[b]{2}{*}{ Characteristics } & \multicolumn{2}{|c|}{$n 889$} & \multicolumn{6}{|c|}{$\mathrm{F}_{2}$-IsoP } & \multicolumn{6}{|c|}{$15-F_{2 t}$ IsoP-M } \\
\hline & $n$ & $\%$ & GM & $95 \% \mathrm{Cl}+$ & uGMD & $95 \% \mathrm{Cl}$ & aGMD & $95 \% \mathrm{Cl}$ & GM & $95 \% \mathrm{Cl} \dagger$ & uGMD & $95 \% \mathrm{Cl}$ & aGMD & $95 \% \mathrm{Cl} \S$ \\
\hline \multicolumn{15}{|c|}{ Healthy eating index $(0-100)$} \\
\hline$<53$ & 213 & $24 \cdot 0$ & 1.54 & $1.42,1.66$ & & Ref. & & Ref. & 0.77 & $0.72,0.82$ & \multicolumn{3}{|c|}{ Ref. } & \multirow{4}{*}{$\begin{array}{l}-0.06,0.11 \\
-0.10,0.10 \\
-0.09,0.14\end{array}$} \\
\hline $53-<63$ & 230 & $25 \cdot 9$ & 1.54 & $1.44,1.64$ & 0.00 & $-0.10,0.09$ & 0.08 & $-0.02,0.18$ & 0.75 & $0.71,0.79$ & -0.03 & $-0.12,0.05$ & 0.02 & \\
\hline $63-<72$ & 223 & $25 \cdot 1$ & 1.35 & $1.27,1.44$ & -0.12 & $-0.22,-0.03$ & 0.06 & $-0.06,0.18$ & 0.68 & $0.64,0.72$ & -0.14 & $-0.22,-0.05$ & 0.00 & \\
\hline$\geq 72$ & 223 & $25 \cdot 1$ & 1.34 & $1 \cdot 26,1.44$ & -0.14 & $-0.23,-0.04$ & 0.13 & $-0.01,0.26$ & 0.66 & $0.62,0.70$ & -0.17 & $-0.25,-0.08$ & 0.03 & \\
\hline \multicolumn{15}{|c|}{ Dietary fruit intake (servings/d) } \\
\hline$<0.6$ & 210 & $23 \cdot 6$ & 1.62 & $1.51,1.73$ & & Ref. & & Ref. & 0.78 & $0.73,0.82$ & \multicolumn{3}{|c|}{ Ref. } & \multirow{4}{*}{$\begin{array}{c}\text { Ref. } \\
-0 .\end{array}$} \\
\hline $0.6-<1.1$ & 212 & 23.9 & 1.51 & $1.40,1.63$ & -0.05 & $-0.15,0.05$ & -0.04 & $-0.15,0.06$ & 0.73 & $0.69,0.78$ & -0.07 & $-0.16,0.02$ & -0.01 & \\
\hline $1 \cdot 1-<2$ & 203 & $22 \cdot 8$ & 1.40 & $1.31,1.48$ & -0.13 & $-0.23,-0.03$ & -0.09 & $-0.22,0.04$ & 0.71 & $0.67,0.76$ & -0.08 & $-0.17,0.01$ & 0.01 & \\
\hline$\geq 2$ & 264 & 29.7 & 1.29 & $1 \cdot 21,1 \cdot 37$ & -0.21 & $-0.31,-0.12$ & -0.13 & $-0.27,0.00$ & 0.65 & $0.62,0.69$ & -0.19 & $-0.27,-0.11$ & -0.01 & \\
\hline \multicolumn{15}{|c|}{ Dietary vegetable intake (servings/d) } \\
\hline$<1.6$ & 202 & $22 \cdot 7$ & 1.63 & $1.51,1.75$ & \multicolumn{4}{|c|}{ Ref. } & 0.75 & $0.71,0.80$ & \multicolumn{3}{|c|}{ Ref. } & \multirow{4}{*}{$\begin{array}{l}9 \\
7 \\
6\end{array}$} \\
\hline $1 \cdot 6-<2 \cdot 7$ & 240 & $27 \cdot 0$ & 1.45 & $1.36,1.55$ & -0.10 & $-0.20,-0.01$ & -0.09 & $-0.20,0.03$ & 0.76 & $0.72,0.80$ & 0.01 & $-0.08,0.09$ & 0.09 & \\
\hline $2 \cdot 7-<4 \cdot 3$ & 221 & 24.9 & 1.40 & $1.31,1.49$ & -0.15 & $-0.25,-0.05$ & -0.09 & $-0.24,0.06$ & 0.67 & $0.64,0.71$ & -0.11 & $-0.20,-0.03$ & 0.05 & \\
\hline$\geq 4 \cdot 3$ & 226 & $25 \cdot 4$ & $1 \cdot 31$ & $1.22,1.40$ & -0.22 & $-0.32,-0.12$ & -0.05 & $-0.23,0.13$ & 0.67 & $0.63,0.71$ & -0.13 & $-0.22,-0.04$ & 0.12 & \\
\hline \multicolumn{15}{|c|}{ Dietary $\beta$-carotene intake $(\mu \mathrm{g} / \mathrm{d})$} \\
\hline$<2427 \cdot 2$ & 222 & $25 \cdot 0$ & 1.63 & $1.52,1.74$ & & Ref. & & Ref. & 0.80 & $0.75,0.84$ & \multicolumn{2}{|r|}{ Ref. } & \multicolumn{2}{|r|}{ Ref. } \\
\hline $2427 \cdot 2-<4106 \cdot 1$ & 222 & $25 \cdot 0$ & 1.49 & $1.38,1.60$ & -0.07 & $-0.17,0.02$ & 0.01 & $-0.11,0.13$ & 0.75 & $0.71,0.80$ & -0.06 & $-0.15,0.02$ & -0.04 & $-0.14,0.06$ \\
\hline $4106 \cdot 1-<6942 \cdot 3$ & 222 & $25 \cdot 0$ & 1.42 & $1.32,1.52$ & -0.13 & $-0.23,-0.04$ & -0.02 & $-0.18,0.13$ & 0.67 & $0.64,0.72$ & -0.18 & $-0.27,-0.10$ & -0.13 & $-0.26,-0.01$ \\
\hline$\geq 6942 \cdot 3$ & 223 & $25 \cdot 1$ & 1.25 & $1.17,1.33$ & -0.26 & $-0.36,-0.16$ & -0.13 & $-0.31,0.05$ & 0.64 & $0.60,0.68$ & -0.22 & $-0.31,-0.14$ & -0.16 & $-0.31,-0.01$ \\
\hline \multicolumn{15}{|c|}{ Dietary vitamin C intake $(\mathrm{mg} / \mathrm{d})$} \\
\hline$<55.3$ & 221 & 24.9 & 1.60 & $1.48,1.72$ & & Ref. & & Ref. & 0.77 & $0.73,0.82$ & \multicolumn{2}{|r|}{ Ref. } & \multicolumn{2}{|r|}{ Ref. } \\
\hline $55 \cdot 3-<84 \cdot 1$ & 223 & $25 \cdot 1$ & 1.48 & $1.39,1.58$ & -0.06 & $-0.16,0.03$ & 0.00 & $-0.11,0.11$ & 0.75 & $0.71,0.79$ & -0.04 & $-0.13,0.04$ & 0.02 & $-0.07,0.11$ \\
\hline $84.1-<121 \cdot 9$ & 222 & $25 \cdot 0$ & 1.38 & $1.29,1.48$ & -0.14 & $-0.24,-0.04$ & -0.01 & $-0.14,0.12$ & 0.67 & $0.63,0.71$ & -0.15 & $-0.23,-0.06$ & -0.03 & $-0.13,0.08$ \\
\hline$\geq 121 \cdot 9$ & 223 & $25 \cdot 1$ & $1 \cdot 30$ & $1.22,1.39$ & -0.20 & $-0.30,-0.11$ & -0.04 & $-0.20,0.12$ & 0.67 & $0.63,0.72$ & -0.14 & $-0.22,-0.06$ & -0.01 & $-0.14,0.12$ \\
\hline \multicolumn{15}{|c|}{ Dietary vitamin $E$ intake $(\mathrm{mg} / \mathrm{d})$} \\
\hline$<5 \cdot 6$ & 217 & $24 \cdot 4$ & 1.54 & $1.43,1.65$ & & Ref. & & Ref. & 0.77 & $0.73,0.82$ & \multicolumn{2}{|r|}{ Ref. } & \multicolumn{2}{|r|}{ Ref. } \\
\hline $5 \cdot 6-<7 \cdot 6$ & 225 & $25 \cdot 3$ & 1.44 & $1.33,1.55$ & -0.07 & $-0.17,0.03$ & -0.03 & $-0.15,0.08$ & 0.70 & $0.66,0.74$ & -0.11 & $-0.19,-0.02$ & -0.09 & $-0.18,0.01$ \\
\hline $7.6-<10.1$ & 222 & $25 \cdot 0$ & 1.46 & $1.37,1.57$ & -0.06 & $-0.15,0.04$ & -0.04 & $-0.17,0.09$ & 0.71 & $0.67,0.76$ & -0.08 & $-0.17,0.00$ & -0.10 & $-0.21,0.01$ \\
\hline$\geq 10.1$ & 225 & $25 \cdot 3$ & 1.33 & $1.25,1.41$ & -0.16 & $-0.26,-0.06$ & -0.13 & $-0.29,0.03$ & 0.67 & $0.63,0.72$ & -0.14 & $-0.22,-0.05$ & -0.18 & $-0.31,-0.05$ \\
\hline
\end{tabular}

UGMD, univariate geometric mean difference; aGMD, adjusted geometric mean difference; Ref., reference; MET, metabolic equivalent of task. * Geometric mean difference and $95 \% \mathrm{Cl}$ were calculated on the basis of natural logarithm of $\mathrm{F}_{2}-\mathrm{IsoP}$ and $15-\mathrm{F}_{2 \mathrm{t}}$-IsoP-M.

† GM was calculated using the whole sample ( $n$ 889).

‡ Univariate models were restricted to participants without missing values of the covariates and had the same size as adjusted models $(n 856)$. $\S$ aGMD was calculated in multivariable model adjusting for all variables in Table 1. 
Table 2. Association between tea consumption or caffeine intake and urinary 8-iso- $\mathrm{PGF}_{2 a}\left(\mathrm{~F}_{2}-\mathrm{Iso}\right)$ or 2,3-dinor-5,6-dihydro-15-F2t-isoprostane (15- $\mathrm{F}_{2 \mathrm{t}^{-}}$ IsoP-M)*

(Numbers and percentages; geometric means (GM) and $95 \%$ confidence intervals)

\begin{tabular}{|c|c|c|c|c|c|c|c|c|c|c|c|c|c|c|}
\hline \multirow[b]{2}{*}{ Characteristics } & \multicolumn{2}{|c|}{$n 889$} & \multicolumn{6}{|c|}{$\mathrm{F}_{2}$-IsoP } & \multicolumn{6}{|c|}{$15-\mathrm{F}_{2 \mathrm{t}}$-IsoP-M } \\
\hline & $n$ & $\%$ & GM & $95 \% \mathrm{Cl}+$ & uGMD & $95 \% \mathrm{Cl}$ & aGMD & $95 \% \mathrm{Cl} \S$ & GM & $95 \% \mathrm{Cl} \dagger$ & uGMD & $95 \% \mathrm{Cl}$ & aGMD & $95 \% \mathrm{Cl} \S$ \\
\hline \multicolumn{15}{|c|}{ Black tea (cups/week) } \\
\hline 0 & 165 & $18 \cdot 6$ & 1.50 & $1.38,1.63$ & & Ref. & & Ref. & 0.71 & $0.66,0.75$ & & Ref. & & Ref. \\
\hline$<1$ & 311 & $35 \cdot 0$ & 1.39 & $1.32,1.47$ & -0.07 & $-0.17,0.03$ & -0.06 & $-0.15,0.04$ & 0.70 & $0.67,0.74$ & 0.00 & $-0.09,0.08$ & 0.04 & $-0.04,0.12$ \\
\hline $1-<5$ & 187 & $21 \cdot 0$ & 1.38 & $1.28,1.48$ & -0.08 & $-0.19,0.03$ & -0.09 & $-0.20,0.01$ & 0.68 & $0.64,0.73$ & -0.04 & $-0.13,0.06$ & -0.01 & $-0.10,0.08$ \\
\hline$\geq 5$ & 221 & 24.9 & 1.51 & $1.40,1.62$ & 0.01 & $-0.09,0.12$ & 0.01 & $-0.10,0.11$ & 0.76 & $0.71,0.81$ & 0.08 & $-0.02,0.17$ & 0.10 & $0.02,0.19$ \\
\hline Missing & 5 & 0.5 & 1.62 & $0.81,3.24$ & & & & & 0.63 & $0.34,1.15$ & & & & \\
\hline \multicolumn{15}{|c|}{ Green tea (cups/week) } \\
\hline 0 & 408 & $45 \cdot 9$ & 1.47 & $1.40,1.55$ & & Ref. & & Ref. & 0.72 & $0.69,0.75$ & & Ref. & & Ref. \\
\hline$<1$ & 315 & $35 \cdot 4$ & 1.45 & $1.37,1.54$ & 0.00 & $-0.08,0.07$ & 0.03 & $-0.05,0.10$ & 0.72 & $0.68,0.76$ & 0.00 & $-0.07,0.06$ & 0.05 & $-0.01,0.12$ \\
\hline $1-<5$ & 96 & $10 \cdot 8$ & 1.29 & $1.17,1.43$ & -0.12 & $-0.24,-0.01$ & -0.03 & $-0.14,0.08$ & 0.66 & $0.61,0.72$ & -0.09 & $-0.19,0.01$ & 0.01 & $-0.09,0.10$ \\
\hline$\geq 5$ & 67 & $7 \cdot 6$ & 1.41 & $1.24,1.60$ & -0.04 & $-0.18,0.09$ & 0.06 & $-0.07,0.19$ & 0.70 & $0.64,0.78$ & -0.02 & $-0.14,0.10$ & 0.09 & $-0.02,0.20$ \\
\hline Missing & 3 & 0.3 & 1.17 & $0.24,5.77$ & & & 0.67 & $0 \cdot 21,2 \cdot 16$ & & & & & & \\
\hline \multicolumn{15}{|c|}{ Caffeine intake $(\mathrm{mg} / \mathrm{d})$} \\
\hline$<33.9$ & 222 & $25 \cdot 0$ & 1.42 & $1.33,1.52$ & & Ref. & & Ref. & 0.67 & $0.64,0.71$ & & Ref. & & Ref. \\
\hline $33.9-<111.2$ & 223 & $25 \cdot 1$ & 1.46 & $1.36,1.57$ & 0.03 & $-0.07,0.13$ & 0.03 & $-0.07,0.13$ & 0.74 & $0.69,0.79$ & 0.09 & $0.00,0.17$ & 0.09 & $0.01,0.17$ \\
\hline $111 \cdot 2-<205 \cdot 2$ & 221 & 24.9 & 1.38 & $1.29,1.48$ & -0.03 & $-0.13,0.06$ & -0.02 & $-0.11,0.08$ & 0.69 & $0.65,0.74$ & 0.03 & $-0.06,0.11$ & 0.05 & $-0.03,0.13$ \\
\hline$\geq 205 \cdot 2$ & 223 & $25 \cdot 0$ & 1.49 & $1.39,1.59$ & 0.03 & $-0.07,0.12$ & 0.01 & $-0.09,0.11$ & 0.75 & $0.71,0.79$ & $0 \cdot 10$ & $0.02,0.18$ & 0.08 & $0.00,0.16$ \\
\hline \multicolumn{15}{|c|}{ Black tea (cups/week)\| } \\
\hline 0 & & & & & & & & Ref. & & & & & & Ref. \\
\hline$<1$ & & & & & & & -0.05 & $-0.15,0.04$ & & & & & 0.03 & $-0.05,0.11$ \\
\hline $1-<5$ & & & & & & & -0.09 & $-0.20,0.02$ & & & & & -0.02 & $-0.11,0.07$ \\
\hline$\geq 5$ & & & & & & & 0.01 & $-0.10,0.12$ & & & & & 0.08 & $-0.01,0.17$ \\
\hline \multicolumn{15}{|c|}{ Green tea (cups/week)\| } \\
\hline 0 & & & & & & & & Ref. & & & & & & Ref. \\
\hline$<1$ & & & & & & & 0.03 & $-0.05,0.10$ & & & & & 0.05 & $-0.02,0.11$ \\
\hline $1-<5$ & & & & & & & -0.03 & $-0.15,0.08$ & & & & & -0.01 & $-0.10,0.09$ \\
\hline$\geq 5$ & & & & & & & 0.05 & $-0.08,0.19$ & & & & & 0.07 & $-0.04,0.18$ \\
\hline
\end{tabular}

uGMD, univariate geometric mean difference; aGMD, adjusted geometric mean difference; Ref., reference.

* Geometric mean difference and $95 \% \mathrm{Cl}$ were calculated on the basis of natural logarithm of $\mathrm{F}_{2}$-IsoP and $15-\mathrm{F}_{2 t}-\mathrm{IsoP}_{\mathrm{I}} \mathrm{M}$.

† GM was calculated using the whole sample ( $n$ 889).

‡ Univariate models were restricted to participants without missing values of the covariates (black tea: $n$ 858, green tea: $n 861$, caffeine: $n$ 863).

$\S$ The multivariable model adjusted for age, race, smoking status, BMI, physical activity, household income, education level, energy intake, healthy eating index, dietary fruit, vegetable, $\beta$-carotene, vitamin $C$ and vitamin $\mathrm{E}$ intake (black tea: $n$ 858, green tea: $n$ 861, caffeine: $n 863$ ).

\| The multivariable model additionally adjusted for caffeine intake.

consumption and urinary $\mathrm{F}_{2}$-IsoP or $15-\mathrm{F}_{2 \mathrm{t}}$-IsoP-M were not modified by overweight (online Supplementary Table S1) or regular coffee consumption (online Supplementary Table S2).

\section{Discussion}

Our analysis did not provide support for an inverse association between dietary tea consumption and urinary $\mathrm{F}_{2}-\mathrm{IsoP}$ or $15-\mathrm{F}_{2 \mathrm{t}^{-}}$ IsoP-M, high-quality biomarkers of oxidative stress. Green tea consumption was not associated with either $\mathrm{F}_{2}$-IsoP or $15-\mathrm{F}_{2 \mathrm{t}}{ }^{-}$ IsoP-M. Black tea consumption was not associated with $\mathrm{F}_{2}$-IsoP; however, drinking at least 5 cups of black tea/week (compared with none) was associated with higher $15-\mathrm{F}_{2 \mathrm{t}}-\mathrm{IsoP}-\mathrm{M}$ concentrations before adjustment for caffeine.

Clinical studies have found an inverse association between tea consumption and oxidative stress ${ }^{(25-27,50)}$. For example, by observing nineteen people in a 5-d experimental study, Stote et $a l .{ }^{(50)}$ found that green tea consumption could lower plasma levels of $\mathrm{F}_{2}$-IsoP. However, subjects in this study consumed a higher level of tea (e.g. 2 servings of green tea/d for 5 d) than was commonly consumed in our population-based sample of the US women. In addition, this study enroled only nineteen obese people at high risk of insulin resistance, which may have compromised the generalisability of their outcomes.

A cross-sectional epidemiological study ${ }^{(12)}$ of 845 Chinese women observed an almost null association between any tea drinking and urinary levels of $\mathrm{F}_{2}$-IsoP (geometric mean: never drinker: 1.62 , ever drinker: $1.65, P=0.72$ ) and $15-\mathrm{F}_{2 \mathrm{t}}$-IsoP-M (geometric mean: never drinker: 0.56, ever drinker: 0.61, $P=0.06$ ) after adjustment for age, education, occupation, smoking, BMI, multivitamin supplement use, fruit and vegetable intakes, plasma total carotenoids, tocopherols and retinol, assay batch and urinary tea polyphenols. However, the ever/never analysis did not consider level of consumption or potential difference between black and green tea ${ }^{(12)}$. Green and black tea differ in concentrations of polyphenols (e.g. EGCG) and caffeine ${ }^{(51,52)}$. For example, green tea has a higher level of EGCG compared with black tea ${ }^{(53)}$, while black tea contains more caffeine ${ }^{(51)}$. In addition, a previous study measuring total phenol levels and antioxidant capacity of tea products sold in the USA found green tea had a higher antioxidant capacity than black tea of the same volume (436 v. $239 \mathrm{mg}$ vitamin C equivalents per serving) ${ }^{(54)}$. These suggest analysis pooling all types of tea in to one category may obscure meaningful variation. 
$15-\mathrm{F}_{2 \mathrm{t}}$-IsoP-M is the metabolite of $\mathrm{F}_{2}$-IsoP under $\beta$-oxidation $^{(12)}$. Both black and green tea contain EGCG and caffeine which have been found to facilitate $\beta$-oxidation on the basis of laboratory evidence ${ }^{(55,56)}$. The suggested positive associations between black tea and caffeine with $15-\mathrm{F}_{2 \mathrm{t}}$-IsoP-M, but not $\mathrm{F}_{2^{-}}$ IsoP, may be due, in part, to related increases in $\beta$-oxidation pathways. We did not observe an association between green tea and $15-\mathrm{F}_{2 \mathrm{t}}$-IsoP-M; however, there were few high-level green tea consumers in our analysis.

Our results regarding the association between caffeine and $15-\mathrm{F}_{2 \mathrm{t}}$-IsoP-M were similar to an experimental study ${ }^{(57)}$ that assigned twenty participants caffeine $(5 \mathrm{mg} / \mathrm{kg})$ or placebo before physical exercise and observed a positive association between caffeine and plasma MDA using blood samples collected immediately after exercise. However, other experimental studies have reported inverse associations between caffeine intake and biomarkers of oxidative stress using other caffeine dosages or different biomarkers of antioxidant activity (e.g. plasma glutathione) or oxidative stress (e.g. 8-hydroxydeoxyguanosine $)^{(58,59)}$. Due to the different study design, biomarkers used for analysis and divergent findings to date, the association between caffeine and oxidative stress deserves further investigation.

Strengths of our study included the use of a general population sample, the clear categorisation of tea type and detailed information on socio-demographic and lifestyle factors for statistical adjustment. Particularly, using a population-based sample can better reflect real-world tea consumption pattern as compared with high-level tea assignment in experimental studies. The use of urinary $\mathrm{F}_{2}$-IsoP provided a stable biomarker of lipid peroxidation. A previous biochemical study has shown that plasma stored appropriately for at least 10 years has $\mathrm{F}_{2}$-IsoP levels similar to freshly prepared samples ${ }^{(13)}$. Given that artefactual generation of $\mathrm{F}_{2}$-IsoP through autoxidation of lipids only occurs in plasma and not in urine ${ }^{(12,13)}$, our samples were likely to be equally or more stable. In some previous studies, MDA was used as the biomarker of oxidative stress ${ }^{(25,27)}$; however, MDA is more affected by dietary lipid consumption ${ }^{(60)}$ and can be generated from nonlipid sources such as bile pigments ${ }^{(61)}$, which may cause measurement error. 8-Hydroxy-2'-deoxyguanosine (8-OHdG) is another biomarker of oxidative stress which is an end product of non-enzymatic DNA oxidation ${ }^{(62)}$. However, levels of $8-\mathrm{OHdG}$ can be influenced by DNA repair capacity which makes 8-OHdG an indicator of the combined effects of oxidative stress-associated damage and DNA repair capability. Furthermore, a previous study of ten healthy volunteer suggests that there is not a significant diurnal variation in urinary $\mathrm{F}_{2}$-IsoP ${ }^{(63)}$, whereas many studies suggest that diurnal variation in urinary $8-\mathrm{OHdG}$ is substantial $^{(62,64,65)}$. These characteristics make urinary $\mathrm{F}_{2}$-IsoP a more desirable biomarker of oxidative stress.

Our study also has some limitations. Our sample included only premenopausal women, which may compromise the external validity for men, older women or individuals with specific medical conditions. Also, tea consumption was measured by retrospective self-report, and non-differential measurement error could be introduced. Duration of tea consumption was not available for analysis, and the antioxidant potential of green and black tea sources was not directly measured. Finally, our study is a cross-sectional analysis with one-time urinary sampling and two biomarkers of oxidative, which makes it inappropriate for casual interpretation.

Our study contributes real-world data regarding associations between tea consumption and oxidative stress. In our study, we did not observe an inverse association between green or black tea consumption and urinary $\mathrm{F}_{2}$-IsoP. Additional studies with detailed information on the timing and duration of tea consumption, and additional measures of oxidative stress, may be warranted to inform the use of antioxidant products.

\section{Acknowledgements}

The authors appreciate the helpful comments of Dr Thomas Joost Van't Erve.

This work was supported, in part, by the National Institute of Environmental Health Sciences (Z01 ES044005) and the Avon Foundation (02-2012-085).

D. Z., H. B. N. and D. P. S. designed the study. D. P. S. and G. L. M. conducted the study. D. Z. conducted statistical analysis. All the authors contributed to manuscript writing and revision. All the authors read and approved the final version of the manuscript.

None of the authors had any conflict of interest.

\section{Supplementary material}

For supplementary material/s referred to in this article, please visit https://doi.org/10.1017/S0007114518003732

\section{References}

1. Katiyar SK \& Mukhtar H (1997) Tea antioxidants in cancer chemoprevention. J Cell Biochem Suppl 27, 59-67.

2. Junqueira VB, Barros SB, Chan SS, et al. (2004) Aging and oxidative stress. Mol Aspects Med 25, 5-16.

3. Roncevic M (2007) Encyclopedia of stress. Libr J 132, 104-107.

4. Vaziri ND \& Rodriguez-Iturbe B (2006) Mechanisms of disease: oxidative stress and inflammation in the pathogenesis of hypertension. Nat Clin Pract Nephrol 2, 582-593.

5. Kryston TB, Georgiev AB, Pissis P, et al. (2011) Role of oxidative stress and DNA damage in human carcinogenesis. Mutat Res 711, 193-201.

6. Reuter S, Gupta SC, Chaturvedi MM, et al. (2010) Oxidative stress, inflammation, and cancer: how are they linked? Free Radic Biol Med 49, 1603-1616.

7. Coluzzi E, Colamartino M, Cozzi R, et al. (2014) Oxidative stress induces persistent telomeric DNA damage responsible for nuclear morphology change in mammalian cells. PLOS ONE 9, e110963.

8. Sosa V, Moline T, Somoza R, et al. (2013) Oxidative stress and cancer: an overview. Ageing Res Rev 12, 376-390.

9. Nunomura A, Castellani RJ, Zhu X, et al. (2006) Involvement of oxidative stress in Alzheimer disease. J Neuropathol Exp Neurol 65, 631-641.

10. Wu Y, Tang L \& Chen B (2014) Oxidative stress: implications for the development of diabetic retinopathy and antioxidant therapeutic perspectives. Oxid Med Cell Longev 2014, 752387.

11. Milne GL, Musiek ES \& Morrow JD (2005) $\mathrm{F}_{2}$-isoprostanes as markers of oxidative stress in vivo: an overview. Biomarkers 10, Suppl. 1, S10-S23. 
12. Dorjgochoo T, Gao YT, Chow WH, et al. (2012) Major metabolite of $\mathrm{F}_{2}$-isoprostane in urine may be a more sensitive biomarker of oxidative stress than isoprostane itself. Am J Clin Nutr 96, 405-414.

13. Van't Erve TJ, Lih FB, Jelsema C, et al. (2016) Reinterpreting the best biomarker of oxidative stress: the 8-iso-prostaglandin F2alpha/prostaglandin F2alpha ratio shows complex origins of lipid peroxidation biomarkers in animal models. Free Radic Biol Med 95, 65-73.

14. Rossner P Jr, Gammon MD, Terry MB, et al. (2006) Relationship between urinary $15-\mathrm{F}_{2 \mathrm{t}}$-isoprostane and 8oxodeoxyguanosine levels and breast cancer risk. Cancer Epidemiol Biomarkers Prev 15, 639-644.

15. Schwedhelm E, Bartling A, Lenzen H, et al. (2004) Urinary 8-iso-prostaglandin F2alpha as a risk marker in patients with coronary heart disease: a matched case-control study. Circulation 109, 843-848.

16. van 't Erve TJ, Kadiiska MB, London SJ, et al. (2017) Classifying oxidative stress by $\mathrm{F}_{2}$-isoprostane levels across human diseases: a meta-analysis. Redox Biol 12, 582-599.

17. Lin JK, Liang YC \& Lin-Shiau SY (1999) Cancer chemoprevention by tea polyphenols through mitotic signal transduction blockade. Biochem Pharmacol 58, 911-915.

18. Yang WS, Wang WY, Fan WY, et al. (2014) Tea consumption and risk of type 2 diabetes: a dose-response meta-analysis of cohort studies. Br J Nutr 111, 1329-1339.

19. Lambert JD (2013) Does tea prevent cancer? Evidence from laboratory and human intervention studies. Am J Clin Nutr $\mathbf{9 8}$, 1667S-1675s

20. Arab L, Khan F \& Lam H (2013) Tea consumption and cardiovascular disease risk. Am J Clin Nutr 98, 1651S-1659S

21. Chen D, Wan SB, Yang H, et al. (2011) EGCG, green tea polyphenols and their synthetic analogs and prodrugs for human cancer prevention and treatment. Adv Clin Chem $\mathbf{5 3}$, $155-177$.

22. Scalbert A, Johnson IT \& Saltmarsh M (2005) Polyphenols: antioxidants and beyond. Am J Clin Nutr 81, 215S-217S.

23. Cavet ME, Harrington KL, Vollmer TR, et al. (2011) Antiinflammatory and anti-oxidative effects of the green tea polyphenol epigallocatechin gallate in human corneal epithelial cells. Mol Vis 17, 533-542.

24. Tipoe GL, Leung TM, Hung MW, et al. (2007) Green tea polyphenols as an anti-oxidant and anti-inflammatory agent for cardiovascular protection. Cardiovasc Hematol Disord Drug Targets 7, 135-144.

25. Basu A, Sanchez K, Leyva MJ, et al. (2010) Green tea supplementation affects body weight, lipids, and lipid peroxidation in obese subjects with metabolic syndrome. J Am Coll Nutr 29, 31-40.

26. Bogdanski P, Suliburska J, Szulinska M, et al. (2012) Green tea extract reduces blood pressure, inflammatory biomarkers, and oxidative stress and improves parameters associated with insulin resistance in obese, hypertensive patients. Nutr Res $\mathbf{3 2}$, 421-427.

27. Klaunig JE, Xu Y, Han C, et al. (1999) The effect of tea consumption on oxidative stress in smokers and nonsmokers. Proc Soc Exp Biol Med 220, 249-254.

28. Hakim IAGL, Harris R, Cordova CA, et al. (2017) Modulation of oxidative damage by green and black tea: role of smoking and gender in a randomized trial. J Nutr Food Sci 7, 633.

29. Sandler DP, Hodgson ME, Deming-Halverson SL, et al. (2017) The Sister Study cohort: baseline methods and participant characteristics. Environ Health Perspect 125, 127003.

30. Parks CG, Miller DB, McCanlies EC, et al. (2009) Telomere length, current perceived stress, and urinary stress hormones in women. Cancer Epidemiol Biomarkers Prev 18, 551-560.

31. Milne GL, Sanchez SC, Musiek ES, et al. (2007) Quantification of $\mathrm{F}_{2}$-isoprostanes as a biomarker of oxidative stress. Nat Protoc 2, 221-226.

32. Morrow JD \& Roberts LJ 2nd (1999) Mass spectrometric quantification of $\mathrm{F}_{2}$-isoprostanes in biological fluids and tissues as measure of oxidant stress. Methods Enzymol 300, 3-12.

33. Morales CR, Terry ES, Zackert WE, et al. (2001) Improved assay for the quantification of the major urinary metabolite of the isoprostane 15-F(2t)-Isoprostane (8-iso-PGF(2alpha)) by a stable isotope dilution mass spectrometric assay. Clin Chim Acta 314, 93-99.

34. Milne GL, Gao B, Terry ES, et al. (2013) Measurement of $\mathrm{F}_{2}$-isoprostanes and isofurans using gas chromatographymass spectrometry. Free Radic Biol Med 59, 36-44.

35. Anderson C, Milne GL, Sandler DP, et al. (2016) Oxidative stress in relation to diet and physical activity among premenopausal women. Br J Nutr 116, 1416-1424.

36. Lin CJ, DeRoo LA, Jacobs SR, et al. (2012) Accuracy and reliability of self-reported weight and height in the Sister Study. Public Health Nutr 15, 989-999.

37. Block G, Hartman AM, Dresser CM, et al. (1986) A data-based approach to diet questionnaire design and testing. Am J Epidemiol 124, 453-469.

38. Nutrient Analysis Data Overview (2008) https://docs. sssepistudies.org/studies/sisterstudy/Baseline/NA_placeholder.pdf (accessed February 2018).

39. Mitchell DC, Knight CA, Hockenberry J, et al. (2014) Beverage caffeine intakes in the US. Food Chem Toxicol 63, 136-142.

40. United States Department of Agriculture, Agricultural Research Service (2018) USDA Food Composition Databases. https://ndb.nal.usda.gov/ndb/search/list?qlookup=19902 (accessed September 2018).

41. Ainsworth B (2002) The Compendium of Physical Activities Tracking Guide. Columbia, SC: Prevention Research Center, Norman J Arnold School of Public Health, University of South Carolina.

42. Craig CL, Marshall AL, Sjostrom M, et al. (2003) International Physical Activity Questionnaire: 12-country reliability and validity. Med Sci Sports Exerc 35, 1381-1395.

43. World Health Organization (2017) Body mass index - BMI. http://www.euro.who.int/en/health-topics/disease-prevention/ nutrition/a-healthy-lifestyle/body-mass-index-bmi (accessed December 2017).

44. Willett WC, Howe GR \& Kushi LH (1997) Adjustment for total energy intake in epidemiologic studies. Am J Clin Nutr $\mathbf{6 5}$, 1220S-1228S; discussion 1229S-1231S.

45. Devasagayam TP, Kamat JP, Mohan H, et al. (1996) Caffeine as an antioxidant: inhibition of lipid peroxidation induced by reactive oxygen species. Biochim Biophys Acta 1282, 63-70.

46. Shi X, Dalal NS \& Jain AC (1991) Antioxidant behaviour of caffeine: efficient scavenging of hydroxyl radicals. Food Chem Toxicol 29, 1-6.

47. Nikitina D, Chen Z, Vallis K, et al. (2015) Relationship between caffeine and levels of DNA repair and oxidative stress in women with and without a BRCA1 mutation. J Nutrigenet Nutrigenomics $\mathbf{8}, 174-184$.

48. Rothman KJ (1990) No adjustments are needed for multiple comparisons. Epidemiology 1, 43-46.

49. Savitz DA \& Olshan AF (1998) Describing data requires no adjustment for multiple comparisons: a reply from Savitz and Olshan. Am J Epidemiol 147, 813-814; discussion 815.

50. Stote KS, Clevidence BA, Novotny JA, et al. (2012) Effect of cocoa and green tea on biomarkers of glucose regulation, oxidative stress, inflammation and hemostasis in obese adults at risk for insulin resistance. Eur J Clin Nutr 66, 1153-1159. 
51. Henning SM, Niu Y, Lee NH, et al. (2004) Bioavailability and antioxidant activity of tea flavanols after consumption of green tea, black tea, or a green tea extract supplement. Am J Clin Nutr 80, 1558-1564.

52. Stoner GD \& Mukhtar H (1995) Polyphenols as cancer chemopreventive agents. J Cell Biochem 22, 169-180.

53. Lee KW, Lee HJ \& Lee CY (2002) Antioxidant activity of black tea $v$. green tea. $J$ Nutr $\mathbf{1 3 2}, 785$; author reply 786

54. Lee KW, Lee HJ \& Lee CY (2002) Antioxidant activity of black tea $v$. green tea. $J$ Nutr $\mathbf{1 3 2}, 785$.

55. Rains TM, Agarwal S \& Maki KC (2011) Antiobesity effects of green tea catechins: a mechanistic review. J Nutr Biochem 22, $1-7$.

56. Sinha RA, Farah BL, Singh BK, et al. (2014) Caffeine stimulates hepatic lipid metabolism by the autophagy-lysosomal pathway in mice. Hepatology 59, 1366-1380.

57. Olcina GJ, Timon R, Munoz D, et al. (2008) Caffeine ingestion effects on oxidative stress in a steady-state test at $75 \%$

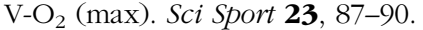

58. Metro D, Cernaro V, Santoro D, et al. (2017) Beneficial effects of oral pure caffeine on oxidative stress. J Clin Transl Endocrinol 10, 22-27.

59. Zeraatpishe A, Malekirad AA, Nik-Kherad J, et al. (2015) The effects of caffeine supplements on exercise-induced oxidative damages. Asian J Sports Med 6, e23023.
60. Richelle M, Turini ME, Guidoux R, et al. (1999) Urinary isoprostane excretion is not confounded by the lipid content of the diet. FEBS Lett 459, 259-262.

61. Janero DR (1990) Malondialdehyde and thiobarbituric acid-reactivity as diagnostic indices of lipid peroxidation and peroxidative tissue injury. Free Radic Biol Med 9, $515-540$

62. Il'yasova D, Scarbrough P \& Spasojevic I (2012) Urinary biomarkers of oxidative status. Clin Chim Acta $\mathbf{4 1 3}$, 1446-1453

63. Helmersson J \& Basu S (1999) $\mathrm{F}_{2}$-isoprostane excretion rate and diurnal variation in human urine. Prostaglandins Leukotrienes Essent Fatty Acids 61, 203-205.

64. Sabatini L, Barbieri A, Tosi M, et al. (2005) A method for routine quantitation of urinary 8-hydroxy-2'-deoxyguanosine based on solid-phase extraction and micro-highperformance liquid chromatography/electrospray ionization tandem mass spectrometry. Rapid Commun Mass Spectrom 19, $147-152$.

65. Pilger A, Ivancsits S, Germadnik D, et al. (2002) Urinary excretion of 8-hydroxy-2'-deoxyguanosine measured by highperformance liquid chromatography with electrochemical detection. J Chromatogr B Analyt Technol Biomed Life Sci 778, 393-401. 